\title{
Synchronization of Kuramoto Oscillators with Non-identical Natural Frequencies: a Quantum Dynamical Decoupling Approach
}

\author{
Zhifei Zhang, Alain Sarlette and Zhihao Ling
}

\begin{abstract}
This paper proposes a method to counter the drift associated to unknown non-identical natural frequencies in the Kuramoto model of coupled oscillators. Inspired by the quantum dynamical decoupling technique, it builds on a time-varying variant of the dynamics to effectively bring the oscillator phases closer to the same value. This allows effective synchronization despite arbitrarily large differences in natural frequencies. For two agents admitting instantaneous position exchanges, we exactly compute how the relative phase converges to a stable periodic fixed point. The latter tends to zero when the dynamics switches at a faster rate. With continuous state evolutions, using a related dynamic controller instead of instantaneous jumps, we show with a Lyapunov function that exact phase synchronization is obtained. We generalize the method to multiple oscillators with instantaneous state exchanges, that can be implemented by cycling through a predefined or random sequence of exchanges. Simulation results illustrate the effectiveness of the algorithms.
\end{abstract}

\section{INTRODUCTION}

The Kuramoto model of coupled oscillators has attracted much interest in chemistry [1], biology [2], as well as physics and engineering [3], [4]. In a typical control application, it describes the interactions governing the planar heading of networked robotic systems, with the objective to achieve coordinated rotation through limited feedback between neighboring robotic agents; other applications could involve e.g. electronic oscillators. When each agent for some reason features a heterogeneous drift ('natural frequency') in addition to the feedback term, the coupling gain must be larger than some critical value [4], [5] to prevent the oscillators from deviating away. Furthermore, even if the coupled oscillators do converge to a common frequency, the residual constant phase differences between oscillators of different natural frequencies can significantly differ from zero. In a control context, this spurs the introduction of some kind of integral control or other, in order to allow oscillators with arbitrary heterogeneous drifts to synchronize towards small phase differences, even under weak coupling strength. The

\footnotetext{
Zhifei Zhang and Zhihao Ling are with the Key Laboratory of Advanced Control and Optimization for Chemical Processes, Ministry of Education, East China University of Science and Technology, No.130, Meilong Road, Shanghai 200237, China. Zhifei Zhang and Alain Sarlette are with the SYSTeMS research group, Faculty of Engineering and Architecture, Ghent University, Technologiepark Zwijnaarde 914, 9052 Zwijnaarde(Ghent), Belgium. zhifei.zhangeugent.be, zhhlingdecust.edu.cn, alain.sarlette@ugent.be.

This paper presents research results of the Belgian Network DYSCO (Dynamical Systems, Control, and Optimization), funded by the Interuniversity Attraction Poles Programme, initiated by the Belgian State, Science Policy Office. The first author's visit to the SYSTeMS research group is supported by a CSC scholarship, initiated by the China Scholarship Council.
}

present paper shows how the quantum dynamical decoupling approach [6], designed to suppress time-independent but unknown drift in quantum system operators, can be applied to this aim in the classical Kuramoto model.

The key point of quantum dynamical decoupling is to iterate unitary actions that do not commute with the timeindependent drift Hamiltonian, in order to effectively average the drift out in the overall evolution (at least to first order in the time between iterations). For example, drift on a single qubit - which is strictly analogous to rotation around a constant axes on a sphere, called the Bloch sphere can be removed by applying rotations orthogonal to the drift direction; if the drift direction is unknown, then two decoupling directions must be concatenated. There are two possible ways to implement these rotations. Bang-Bang decoupling [6] inserts instantaneous pulses of $\pi$ rotation at discrete times, which simplifies the computation of the resulting evolution. As a more realistic model of experimental settings, Eulerian decoupling [7] adds bounded Hamiltonian controls to the existing dynamics, designed such that in absence of drift the system would evolve smoothly through various states corresponding to Cayley graph vertices of a so-called "decoupling group" of transformations. When large decoupling groups are needed, a random switching sequence can be more effective than deterministic cycling [8]. This is interesting for our distributed network application, where we can thus advantageously dispose of a supervisor.

In the present paper, we show that quantum dynamical decoupling can be adapted to synchronize multiple heterogeneous oscillators into a state with arbitrarily small phase differences. We design both Bang-Bang and Eulerian decoupling strategies to synchronize two oscillators. For the multi-agent case, we consider both deterministic and random Bang-Bang decoupling and show their capacity to effectively average out drift, both in simulations and analytically under an averaging approximation. In future work, we will combine the present results towards Eulerian decoupling for multiple agents. The open-loop control nature of quantum dynamical decoupling could be a practical advantage, in settings where the active controller is meant to correct drifts in a network with existing Kuramoto-like coupling. Indeed, also in our non-quantum multi-agent adaptation, the "jump" actions required by Bang-Bang decoupling might be implementable without knowing the system states, by physically exchanging e.g. subsystem connections to external influences. Our adaptation of Eulerian decoupling however introduces a new dynamical variable in the controller, which must be updated on basis of state measurements and reminds 
a kind of integral controller.

The structure of the paper is as follows. In Section II, we recall the notions of Kuramoto model and quantum dynamical decoupling method, and show how they can be linked. In section III, we present synchronization of two oscillators with Bang-Bang decoupling and Eulerian decoupling. In section IV, Bang-Bang decoupling algorithms for multi-agent synchronization are analyzed and simulated, both with deterministic and random decoupling paths.

\section{GENERAL PRINCIPLE}

\section{A. Kuramoto Model}

The Kuramoto model [1] describes a set of $N$ oscillators whose phases attract each other according to:

$$
\dot{\theta}_{k}=\omega_{k}+\frac{K}{N} \sum_{j=1}^{N} \sin \left(\theta_{j}-\theta_{k}\right), \quad k=1,2, \ldots, N,
$$

where $K$ is the coupling strength and $\omega_{k}$ denotes the natural frequency of oscillator $k$. Variants have been studied where each oscillator $k$ is not coupled to all others, but only to a subset $\mathcal{N}_{k}$ specified by some interconnection graph.

A solution $\theta^{*}(t)$ of (1) is said to be phase-locked or frequency synchronized if and only if

$$
\dot{\theta}_{j}^{*}-\dot{\theta}_{k}^{*}=0, \quad \forall j, k \in 1,2, \ldots, N .
$$

Oscillators are further said to be exactly synchronized or phase synchronized if they are phase-locked with equal value, i.e. $\theta_{j}^{*}=\theta_{k}^{*}$ for all $j, k$. The critical condition indicates that oscillators which evolve according to (1) can achieve frequency synchronization only if $K \geq K_{\text {critical }}=$ $\max _{j, k}\left|\omega_{j}-\omega_{k}\right| / 2$, or equivalently

$$
|\kappa|=\frac{2 K}{\left|\omega_{j}-\omega_{k}\right|} \geq 1 \forall j, k .
$$

If $|\kappa|<1$, then (1) allows no relative equilibrium solution $\left(\frac{d}{d t}\left(\theta_{k}-\theta_{j}\right)=0\right.$ for all $\left.j, k\right)$ and the fast oscillator(s) will overtake the slow oscillator(s) repeatedly. However, even when $|\kappa|>1$ and the oscillators get phase-locked, this might happen with very different phase values. This is the standard deviation from equilibrium necessary to compensate drifts in systems with proportional feedback. In applications where the actual goal is phase synchronization, this deviation can be detrimental and dedicated control techniques should be added to compensate the drift.

\section{B. Dynamical Decoupling}

A closed quantum system evolves as

$$
\frac{d}{d t} x=-i\left(H_{0}+H_{c}\right) x
$$

where $x \in \mathbb{C}^{n}$ is the wave function, and we define the Hermitian matrices $H_{0}=H_{0}^{*}, H_{c}=H_{c}^{*} \in \mathbb{C}^{n \times n}$ as the drift and control Hamiltonians respectively. Here * denotes complex conjugate transpose. Quantum Dynamical Decoupling (DD) is a method to design $H_{c}$ in open loop in order to minimize the effect of an unknown $H_{0}$ on any state $x$ [6]. It designs $H_{c}$ such that, (i) for $H_{0}=0$ the system would undergo a periodic motion of "decoupling" period $T_{c}$, and (ii) the possible effects of a nonzero constant $H_{0}$ on the state at $T_{c}, 2 T_{c}, \ldots$ are averaged out (as much as possible) over this periodic motion.

Explicitly, let $U_{c}(t)$ denote the propagator associated with (4) when $H_{0}=0$. DD identifies a discrete group $\mathcal{G}=\left\{g_{j}\right\} \subset$ $S U(n), j=\{1, \ldots,|\mathcal{G}|\}$ and a corresponding representation $P(g)$ such that

$$
\frac{1}{|\mathcal{G}|} \sum_{j} P\left(g_{j}\right)^{*} H_{0} P\left(g_{j}\right)=\lambda I
$$

a multiple of the identity matrix, for all expected disturbances $H_{0}$. It then divides $T_{c}$ into $M|\mathcal{G}|$ subintervals for some integer $M$, and it implements controls $H_{c}$ such that $U_{c}(t) \sim$ $P\left(g_{j}\right)$ over $M$ subintervals for each $j$, with $U_{c}\left(T_{c}\right)=I$ the identity. This open-loop control rotates the effective impact of the drift $H_{0}$, such that its associated drift propagator $U(t)$ satisfies

$$
\frac{d}{d t} U(t)=-i H_{0}^{\mathrm{r}}(t) U(t)
$$

with $H_{0}^{\mathrm{r}}(t)=U_{c}^{*}(t) H_{0} U_{c}(t)$. The effective Hamiltonian $H_{\text {eff }}$ over $T_{c}$ is defined as:

$$
U\left(T_{c}\right)=e^{-i H_{\mathrm{eff}} T_{c}}=e^{-i\left[\bar{H}^{0}+\bar{H}^{1}+\ldots\right] T_{c}},
$$

where the $\bar{H}^{0}, \bar{H}^{1}, \ldots$ are Magnus expansion terms of order $1,2, \ldots$ In particular,

$$
\bar{H}^{0}=\frac{1}{T_{c}} \int_{0}^{T_{c}} H_{0}^{\mathrm{r}}(u) d u .
$$

The general goal of DD is to get $H_{\text {eff }}$ as close as possible to a multiple of the identity matrix. Indeed then the effect of $H_{0}$ at every $t=T_{c}, 2 T_{c}, \ldots$ is suppressed since $U(t)=\exp ^{i \lambda t}$ with $\lambda \in \mathbb{R}$ does not influence outputs in quantum dynamics [9]. Achieving this ideal $H_{\text {eff }}$ at the first order of the Magnus expansion (8) is directly linked to (5).

- Bang-Bang Decoupling (BBD) lets strong pulses in $H_{c}$ implement discrete jumps, such that $U_{c}(t)=P\left(g_{\ell}\right)$ for $t \in[\ell \Delta,(\ell+1) \Delta)$ and $\ell=0,1, \ldots,|\mathcal{G}|-1$, so $T_{c}=|\mathcal{G}| \Delta$. The corresponding first-order effective error Hamiltonian $\bar{H}^{0}$ is exactly (5). In general the discrete $U_{c}(t)$ facilitate computation and hence design of the corresponding $H_{\text {eff }}$, but in practice they require very strong control Hamiltonians.

- Eulerian Decoupling (ED) uses smooth control Hamiltonians of lower amplitude, and thereby achieves $U_{c}(\ell \Delta)=P\left(g_{k}\right), g_{k} \in \mathcal{G}$ only at discrete time steps [7]. A symmetric canceling of $H_{0}$ at all times can then nevertheless be ensured by constructing the sequence $f:(1,2, \ldots, M|\mathcal{G}|) \rightarrow \mathcal{G}: \ell \rightarrow g_{k}$ as an Eulerian path through a Cayley graph constructed from $M$ generators $f_{1}, \ldots, f_{M}$ of $\mathcal{G}$. Indeed, under the reasonable assumption that the transition associated to a given generator $f_{\alpha}$ is each time implemented with the same control profile $H_{c}^{\alpha}(t)$ of duration $\Delta$, the resulting 
first-order effective error Hamiltonian writes $\bar{H}^{0}=$

$$
\begin{aligned}
& \frac{1}{|\mathcal{G}| M \Delta} \sum_{g_{j} \in \mathcal{G}} P\left(g_{j}\right)^{*}\left(\sum_{\alpha=1}^{M} \int_{0}^{\Delta} U_{c}^{\alpha}(s)^{*} H_{0} U_{c}^{\alpha}(s) d s\right) P\left(g_{j}\right) \\
& =\frac{1}{|\mathcal{G}|} \sum_{g_{j} \in \mathcal{G}} P\left(g_{j}\right)^{*} H_{0}^{\prime} P\left(g_{j}\right) .
\end{aligned}
$$

Both these methods aim at canceling $\bar{H}^{0}$, and hence are efficient mainly for small $T_{c}$.

\section{Dynamical Decoupling on Kuramoto}

We can write the Kuramoto model in the complex plane:

$$
\frac{d}{d t}\left[e^{i \theta_{1}}, \cdots, e^{i \theta_{N}}\right]=\left(H_{0}+H_{K}\right)\left[i e^{i \theta_{1}}, \cdots, i e^{i \theta_{N}}\right],
$$

where $H_{0}=\operatorname{diag}\left(\omega_{1}, \ldots, \omega_{N}\right)$ and $H_{K}=\operatorname{diag}\left(f_{1}, \ldots, f_{N}\right)$ with $f_{k}=\frac{K}{\left|\mathcal{N}_{k}\right|} \sum_{j \in \mathcal{N}_{k}} \sin \left(\theta_{j}-\theta_{k}\right)$. This highlights a dynamical structure similar to a quantum system. Remarkably, also the objectives match, since a drift Hamiltonian proportional to the identity matrix is perfectly transparent for phase synchronization in the Kuramoto model.

A difference is that the state in (10) is restricted to $\left\{x \in \mathbb{C}^{N}:\left|x_{k}\right|=1\right.$ for $\left.k=1, \ldots, N\right\}$, while quantum DD exploits the freedom to move in $\left\{x \in \mathbb{C}^{N}:|x|=1\right\}$. In this paper, we replace the effect of $H_{c}$ by either (BBD) exchanging among subsystems the states or the causes of their natural frequencies, or (EDD) adapting $H_{K}$ with a dynamic controller that adds the missing degrees of freedom to (10). Another novelty w.r.t. standard quantum DD is that we here have to analyze the DD effect on $H_{K}$, a Hamiltonian which we want to keep. An important point is that $H_{K}$ is state-dependent, unlike $H_{0}$, and hence it might be differently affected by the time-varying decoupling dynamics.

\section{TWO-AGENT SYNCHRONIZATION}

While our ultimate goal is to work with multiple agents, we present as a preliminary step how to synchronize two oscillators under BBD and ED because this situation allows a detailed analytic study.

For two oscillators, let $\theta=\theta_{2}-\theta_{1}, \omega=\omega_{2}-\omega_{1}$, the incremental dynamics can be derived as:

$$
\dot{\theta}=-2 K \sin (\theta)+\omega .
$$

No equilibrium of $\theta$ exists for $-1<\kappa=(2 K) / \omega<1$. For $|\kappa|>1$, there is a stable fixed point $\theta_{1}^{*}=\arcsin (1 / \kappa)$ with $\cos \left(\theta_{1}^{*}\right)>0$, and an associated unstable fixed point $\theta_{2}^{*}=\arcsin (1 / \kappa)$ with $\cos \left(\theta_{2}^{*}\right)<0$. When $|\kappa|$ tends to 1 from above, these two fixed points converge towards $\pi / 2$, in fact far from phase synchronization $\theta=0$.

For $N=2$ in (10), decoupling is achieved with a twoelements group $\mathcal{G}=\{e, g\}$, where $P(e)$ is the identity matrix and $P(g)=[0,1 ; 1,0]$. This indeed generates effective drifts $H_{0}=\operatorname{diag}\left[\omega_{1}, \omega_{2}\right]$ and $H_{1}=P(g)^{*} H_{0} P(g)=\operatorname{diag}\left[\omega_{2}, \omega_{1}\right]$, whose sum $\bar{H}^{0}$ is proportional to the identity.

\section{A. Bang-Bang Decoupling}

For BBD of two agents, instantaneous switches $P(g)$ at $k T_{c} / 2, k=1,2, \ldots$ can be implemented by exchanging positions or natural frequency(-inducing fields). For the sake of analysis, these situations are equivalent to changing the sign of relative phase:

$$
\theta\left(t_{+}\right)=-\theta\left(t_{-}\right), \quad t=T_{c} / 2, T_{c}, 3 T_{c} / 2,2 T_{c}, \ldots
$$

where $\theta\left(t_{-}\right)$and $\theta(t+)$ are respectively the states just before and just after a switch at $t$. Since BDD is a periodic operation, we analyze the solution of the system (10),(12) at periodic times $\theta\left(k T_{c}\right)$.

Proposition 1: The relative phase $\theta\left(k T_{c}\right)$ between $N=$ 2 oscillators with non-identical frequencies, evolving under (10), (12) and starting from any point on the circle, except one unstable periodic equilibrium, converges to

$$
\theta^{*}=2 \arctan \left(\frac{1}{\omega}\left(\frac{a}{t_{d}}-\sqrt{\left(\frac{a}{t_{d}}\right)^{2}+\omega^{2}}\right)\right),
$$

where $a=\sqrt{\omega^{2}-(2 K)^{2}}, t_{d}=\tan \left(a \cdot T_{c} / 4\right)$ for $0<|\kappa| \leq$ 1 , and $a=\sqrt{(2 K)^{2}-\omega^{2}}, t_{d}=\tanh \left(a \cdot T_{c} / 4\right)$ for $|\kappa|>1$. Proof: When $0<|\kappa| \leq 1$, the general solution of (11) is $\theta(t)=2 \arctan \left(\frac{2 K-a \cdot \tan (a(C-t) / 2)}{\omega}\right)$, where $C$ depends on initial value $\theta(0)$. After the variable change $x=\omega \tan (\theta / 2)$, this general solution writes $x(t)=2 K-a \cdot \tan (a(C-$ $t) / 2)=2 K-a \frac{\tan (a C / 2)-\tan (a t / 2)}{1+\tan (a C / 2) \tan (a t / 2)}$. Using this expression over intervals $\left(0, T_{c} / 2\right)$ and $\left(T_{c} / 2, T_{c}\right)$, with a switch at the end of each period, a few calculations yield $x\left(T_{c+}\right)=$ $C_{1}-\frac{C_{2}}{C_{3}+x(0)}=f(x(0))$ with constants $C_{3}<C_{1}<0<$ $C_{2}$ respectively defined by $C_{1}=m_{d}-m_{-}, C_{2}=m_{d}^{2}$, $C_{3}=m_{d}-m_{+}$where $m_{-}=2 K-a / \tan \left(a T_{c} / 4\right)$, $m_{+}=2 K+a / \tan \left(a T_{c} / 4\right), m_{d}=\frac{-a^{2}}{4 K}\left(1+1 / \tan ^{2}\left(a T_{c} / 4\right)\right)$. Figure 1 illustrates the corresponding discrete-time map $f(\cdot)$. Since $C_{3}<C_{1}<0$, the line $y=x$ can only cross the upperleft curve of $y=f(x)$. Therefore $x\left((k+1) T_{c}\right)=f\left(x\left(k T_{c}\right)\right)$ has two equilibria $x_{1,2}^{*}=\frac{a}{t_{d}} \pm \sqrt{\left(\frac{a}{t_{d}}\right)^{2}+\omega^{2}}$. The negative one $x_{1}^{*}$ corresponds to (13) and it is locally stable since $\left|f^{\prime}\left(x_{1}^{*}\right)\right|<1$ (see Fig. 1); it directly attracts all points $x \in\left(-\infty, x_{2}^{*}\right)$. Points above $x=-C_{3}>0$ get mapped to negative values and then converge to $x_{1}^{*}$, while points in $\left(x_{2}^{*},-C_{3}\right]$ get mapped to increasingly larger values until they exceed $-C_{3}$ and join the former evolution. In conclusion, all points except $x_{2}^{*}$ converge to $x_{1}^{*}$, as announced.

The calculations for $|\kappa|>1$ are similar and therefore not detailed here.

Remark: For fast swapping agents, that is $T_{c} \rightarrow 0$, we have the approximation $x_{1}^{*} \approx-\frac{1}{2} \frac{t_{d} \cdot \omega^{2}}{a} \approx-\frac{1}{8} T_{c} \omega^{2}$ and $\theta_{1}^{*}=$ $2 \arctan \left(\frac{1}{\omega} x_{1}^{*}\right) \approx-\frac{1}{4} T_{c} \omega$. Thus faster switching drives the final relative phase closer to zero, and this independently of $K-$ in particular even for $|\kappa|<1$. This reminds integral control in linear systems, where the end equilibrium does not depend on the proportional feedback strength. 


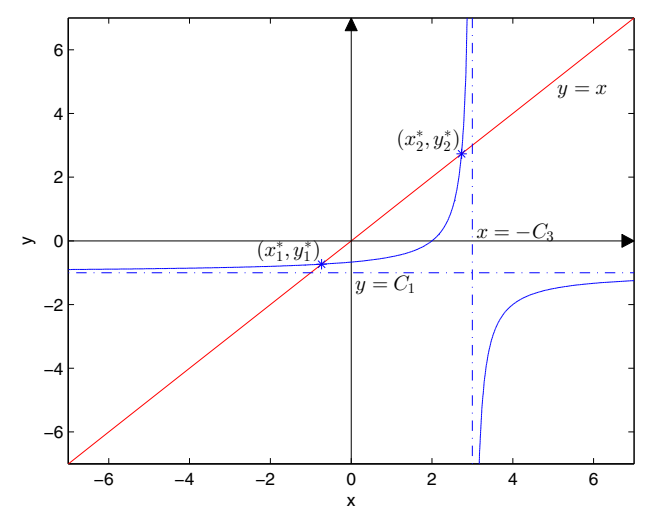

Fig. 1. Function $y=f(x)$ describing the discrete evolution $x((k+$ 1) $\left.T_{c+}\right)=f\left(x\left(k T_{c+}\right)\right)$ of $x=\omega \tan (\theta / 2)$ under (10), (12).

\section{B. Eulerian Decoupling}

Bang-Bang decoupling, requiring instantaneous phase exchanges, has its limitations when applied to physical systems. We therefore propose a synchronization algorithm with continuous decoupling, inspired by EDD. This continuous evolution requires to introduce one more degree of freedom, which will be a dynamic variable of the controller. The smallest setting with EDD is a qubit [7], whose state evolves on the sphere (so-called "Bloch sphere") $S^{2} \subset \mathbb{R}^{3}$. We hence consider that $\theta=\theta_{2}-\theta_{1}$ evolves on the equator of a sphere, and an auxiliary variable is used to model dynamics that is required outside of the equator.

Let $\mathbf{p} \in \mathbb{R}^{3}$ a vector on the unit sphere, denote $\mathbf{z}, \mathbf{x}$ the unit vectors pointing respectively perpendicular to the equator and on the equator at $\theta=0$, and $\times$ the vector product in $\mathbb{R}^{3}$, such that for $\mathbf{y}=\mathbf{z} \times \mathbf{x}$ the vectors $(\mathbf{x}, \mathbf{y}, \mathbf{z})$ form a right-oriented orthonormal frame. Inspired by quantum EDD on the Bloch sphere, we let the drift be a rotation around $\mathbf{z}$ and apply EDD as a constant rotation around $\mathrm{x}$ :

$$
\frac{d}{d t} \mathbf{p}=(\Omega \mathbf{x}+(-2 K \sin \theta+\omega) \mathbf{z}) \times \mathbf{p} .
$$

In this model, $\theta$ is the (azimuth) angle between the $(\mathbf{z}, \mathbf{p})$ plane and the $\mathbf{x}$ axis. It is not defined when $\mathbf{p}$ is parallel to $\mathbf{z}$, but then anyways the corresponding factor $\mathbf{z} \times \mathbf{p}=0$ so it does not matter.

Let $\gamma \in[0, \pi]$ be the (polar) angle between $\mathbf{p}$ and $\mathbf{z}$. Then $\mathbf{p}=[\sin \gamma \cos \theta, \sin \gamma \sin \theta, \cos \gamma]^{T}$ and $d \mathbf{p} / d t=$ $[(2 K \sin \theta-\omega) \sin \gamma \sin \theta,-(2 K \sin \theta-\omega) \sin \gamma \cos \theta-$ $\Omega \cos \gamma, \Omega \sin \gamma \sin \theta]^{T}$. Let $c_{1}$ be the circle through $\mathbf{p}$ and perpendicular to $\mathbf{z}$, and $c_{2}$ the great circle through $\mathbf{p}$ and $\mathrm{z}$ (black circles on Fig.2); the unit vectors tangent to $c_{1}$ and $c_{2}$ are given by $\mathbf{v}_{1}=[-\sin \theta, \cos \theta, 0]^{T}$ and $\mathbf{v}_{2}=$ $[\cos \gamma \cos \theta, \cos \gamma \sin \theta,-\sin \gamma]^{T}$. Projecting $d \mathbf{p} / d t$ onto directions $\mathbf{v}_{1}$ and $\mathbf{v}_{2}$ yields

$$
\begin{aligned}
& \dot{\theta}=\frac{1}{\sin \gamma} \mathbf{v}_{1} \cdot \frac{d \mathbf{p}}{d t}=-(2 K \sin \theta-\omega)-\Omega \cot \gamma \cos \theta, \\
& \dot{\gamma}=\mathbf{v}_{2} \cdot \frac{d \mathbf{p}}{d t}=-\Omega \sin \theta .
\end{aligned}
$$

This is the modified control law that we will apply. Going back to the dynamics of individual agents, we write

$$
\begin{aligned}
& \dot{\theta}_{1}=\left(K \sin \left(\theta_{2}-\theta_{1}\right)+\omega_{1}\right)-\frac{1}{2} \Omega \cot (-\gamma) \cos \left(\theta_{2}-\theta_{1}\right) \\
& \dot{\theta}_{2}=\left(K \sin \left(\theta_{1}-\theta_{2}\right)+\omega_{2}\right)-\frac{1}{2} \Omega \cot \gamma \cos \left(\theta_{1}-\theta_{2}\right)
\end{aligned}
$$

with $\gamma$ an auxiliary variable, shared by both agents. This dynamics features a jump in $\theta$ for the rare trajectories which pass through one of the poles.

For $K=0$, the dynamics $d \mathbf{p} / d t=(\Omega \mathbf{x}+\omega \mathbf{z}) \times \mathbf{p}$ implements a constant rotation rate about the axis $\mathbf{n}=$ $[\Omega, 0, \omega]^{T}$, for which the unit tangent vector is $\mathbf{v}_{\mathbf{3}}$ (blue circle on Fig.2). We now show that the effect added with $K>0$ is to decrease the angle $\phi$ between the vectors $\mathbf{p}$ and $\mathbf{n}$, until $\mathbf{p}$ coincides with $\mathbf{n}$ which means that $\theta=0$. Based on this observation, the convergence of (15) can be summarized as:

Proposition 2: The control law (15) for synchronizing oscillators and countering drift will drive the system asymptotically to the equilibrium $\theta=0, \gamma=\arctan (\Omega / \omega)$, for any starting point except $(\theta(0)=\pi, \gamma(0)=-\arctan (\Omega / \omega))$ which is an unstable equilibrium.

Proof: We propose the Lyapunov function $V=1-\cos \phi$. Projecting $d \mathbf{p} / d t$ onto the direction of $\mathbf{n}$ gives:

$$
\begin{aligned}
\mathbf{n} \cdot \frac{d}{d t} \mathbf{p} & =\left[\begin{array}{c}
\Omega \\
0 \\
\omega
\end{array}\right] \cdot\left[\begin{array}{c}
\Omega \\
0 \\
\omega-K_{p} \sin \theta
\end{array}\right] \times\left[\begin{array}{c}
\sin \gamma \cos \theta \\
\sin \gamma \sin \theta \\
\cos \gamma
\end{array}\right] \\
& =K_{p} \sin \gamma \sin ^{2} \theta \geq 0 .
\end{aligned}
$$

This indicates that the angle between $\mathbf{n}$ and $d \mathbf{p} / d t$ is less than or equal to $90^{\circ}$, thus $\dot{\phi} \leq 0$. Since $0 \leq \phi \leq \pi$, we then have $\dot{V}=\sin \phi \cdot \dot{\phi} \leq 0$. According to LaSalle's invariance principle, the system converges to the largest invariant set where $\dot{V}=0$. In this condition, $\sin (\phi)=0$ corresponds to the global maximum and minimum of $V$, i.e. the unstable initial point and the target equilibrium. Excluding the poles $\sin (\gamma)=0$, the condition $\dot{V}=0$ itself already implies that $\sin (\theta)=0$, i.e. the invariance set must satisfy $\mathbf{y} \cdot \mathbf{p}=0$. Now keeping this condition invariant requires $\mathbf{y} \cdot d \mathbf{p} / d t=0$

$$
\begin{aligned}
& =\left[\begin{array}{l}
0 \\
1 \\
0
\end{array}\right] \cdot\left[\begin{array}{c}
\Omega \\
0 \\
\omega-K_{p} \sin \theta
\end{array}\right] \times\left[\begin{array}{c}
\sin \gamma \cos \theta \\
\sin \gamma \sin \theta \\
\cos \gamma
\end{array}\right] \\
& =\sin \gamma \cos \theta \cdot\left(\omega-K_{p} \sin \theta\right)-\Omega \cos \gamma .
\end{aligned}
$$

This condition in conjunction with $\sin \gamma=0$ requires $\Omega=0$ which does not hold, so the poles cannot belong to the invariant set. In conjunction with $\sin \theta=0$ it reduces to the minimum (target equilibrium) and maximum (excluded unstable starting point) of $V$, for $\theta=0$ and $\theta=\pi$ respectively.

Remark: In (15), the derivative of $\theta$ tends to infinity when $\gamma$ approaches 0 or $\pi$. Although this is not a major problem for the analysis and practical workarounds may be imagined, a more prudent engineer might want to explicitly avoid this singularity. First and foremost, let us clarify that the poles are no accumulation point so in practice almost all trajectories will avoid passing through them. Knowing a bound on the 




Fig. 2. Directions on the sphere for Eulerian decoupling

drift $\|\omega\| \leq \omega_{0}$, constructions which reduce the set of initial states to $V(t=0)<V(\mathbf{p} \in$ poles $)$ would more explicitly ensure that the poles are inaccessible in the future evolution. A better such set is obtained with a small modification of our control law: replace the decoupling $\Omega \mathbf{x}$ by $\Omega \mathbf{x}-\omega_{0} \mathbf{z}$, such that the drift to counter along $\mathbf{z}$ becomes $\left(\omega+\omega_{0}\right)$, possibly larger but certainly positive. Then if we restrict $\theta(0)$ to $[-\pi / 2+\delta, \pi / 2-\delta], \delta>0$ and select $\gamma(0)=\epsilon$ for small enough $\epsilon>0$, we can ensure that $V(t=0)<V(\mathbf{p} \in$ poles $)$. This is different from the analogy with integral control, where we would set $\gamma(0)=\pi / 2$ such that the initial value of the integral term associated to $\theta$ is 0 .

\section{Multi-AGENT SYNCHRONIZATION}

The extension to multiple oscillators involves a new issue besides the selection of an appropriate decoupling group, namely how to implement the decoupling actions over the network. In this section, we first present a strategy where a supervisor would coordinate the decoupling actions, then a strategy with random pairwise permutations. We here consider Bang-Bang decoupling only, and leave for future work an explicit adaptation of Eulerian decoupling with possibly several auxiliary control variables.

\section{A. Deterministic Bang-Bang decoupling}

Suppose that there exists a closed Hamiltonian path, which passes exactly once through all the nodes of the network, and number the agents according to their order on this path. Then the cyclic permutation group generated by $P_{\pi}$ : $P_{\pi}\left[x_{1}, x_{2}, \ldots, x_{N}\right]=\left[x_{2}, \ldots, x_{N}, x_{1}\right]$ can be implemented to counter the drift due to differing natural frequencies. Indeed, since $P_{\pi}^{*} \operatorname{diag}\left(\omega_{1}, \omega_{2}, \ldots, \omega_{N}\right) P_{\pi}=\operatorname{diag}\left(\omega_{2}, \ldots, \omega_{N}, \omega_{1}\right)$, applying $N$ iterations of $P_{\pi}$ over $T_{c}$ yields an effective drift $\frac{1}{N} \sum_{k=0}^{N-1} P_{\pi}^{k *} H_{0} P_{\pi}^{k}=\left(\frac{1}{N} \sum_{k=1}^{N} \omega_{k}\right) \quad I$. We call this method Cyclic Deterministic Bang-Bang Decoupling (CDBBD). If we cannot guarantee that the network has such a closed path, but we only know that it is connected, then it contains a spanning tree $\operatorname{tr}(G)$ with $N-1$ edges. Let $P_{k}$ denote the swap of the two agents linked by edge $k$ of this spanning tree, for $k=1,2, \ldots, N-1$. A recursive argument easily shows that $\prod_{k=1}^{N-1} P_{k}=P_{\pi}$ a cyclic permutation. Hence $N$ repetitions of a cycle of swaps on the $N-1$ edges of the spanning tree has the same effect as CDBBD, only with an enlarged decoupling period covering $N(N-1)$ swaps. We call this approach Swapping Deterministic BangBang Decoupling (SDBBD).

\section{B. Random Bang-Bang decoupling}

We can dispose of the need for a supervisor coordinating the swaps, by letting the decoupling actions take place randomly throughout the network. Such random decoupling has already been investigated in the quantum context [8] with positive results. During a given time interval $\left[0, T_{c}\right]$, we apply decoupling actions at $n$ (more or less) evenly spaced instants. At the $k$-th instant, (not necessarily) independently of previous choices, a set of compatible graph edges whose agents will be swapped is selected randomly from a (more or less) uniform distribution. This can be done in a decentralized way. We denote the corresponding permutation $P_{(k)}$. The effective Hamiltonian over $T$ is then to first order

$$
\bar{H}^{0}=\frac{1}{n} \sum_{j=0}^{n-1} \prod_{k=1}^{j} P_{(k)}^{*} H_{0} P_{(k)} .
$$

The probability for this expression to be $\epsilon$-close to $\lambda I$ converges to 1 as $n$ increases to $\infty$, provided the network is connected [10].

We can finally give the following formal synchronization result for multiple oscillators under Bang-Bang Decoupling.

Proposition 3: Consider $N$ agents with dynamics (10) and applying BBD as described above. The following properties hold for the behavior of the system, in the averaging approximation as $T_{c} \rightarrow 0$. Assume the interconnection graph is connected. We have:

Under SDBBD or CDBBD, if the agents start within a semicircle, then the phases converge to exact synchronization for any $K$ and $\omega_{1}, \ldots, \omega_{N}$.

Under RBBD, for any $\delta \in(0,1)$ and $\varepsilon>0$, there exists $M>0$ such that for any $n>M$, if the agents remain within a semicircle of each other, then the phase is driven into the neighborhood of exact synchronization $\left\|e^{i \theta_{j}}-e^{i \theta_{k}}\right\| \leq \varepsilon$, $\forall j, k=1,2, \ldots, N$, with probability $p \geq \delta$.

Proof: For SDBBD and CDBBD, averaging under decoupling gives the Kuramoto model with equal natural frequencies and a graph that varies in time according to the swapping of agents. It is known that this dynamics converges to phase synchronization, for any time-varying graph, provided the initial conditions are within half a circle (see e.g. [11]). For RBBD we have a similar situation, with decoupling now inducing a randomly varying graph. Any graph sequence would drive the system with equal natural frequencies to exact phase synchronization, when starting within a semicircle. Arguments on random dynamical decoupling [8], [10] can be invoked to bound the effective drift that remains after averaging.

\section{Simulations}

We simulate synchronization under dynamical decoupling with $N=3$ agents with three kinds of switching, CDBBD, 
SDBBD and RBBD. The network for CDBBD is all-toall, while for SDBBD and RBBD there are only two edges as agents 1 and 3 are not directly coupled. We normalize $K=1$ and take drift vector $\boldsymbol{\omega}=[1,3.5,6]^{T}$, initial phase vector $\boldsymbol{\theta}=[0, \pi / 3,2 \pi / 3]^{T}$. The continuous lines show the phase evolutions without dynamical decoupling, which clearly diverge with this parameter choice. Figure 3 shows the evolution of phases under CDBBD, applying $P_{\pi}:(1,2,3) \mapsto(2,3,1)$ every 0.25 s for a decoupling period $T_{c}=0.75 \mathrm{~s}$. We observe that after convergence, the relative phases actually follow an evolution with a shorter period $T_{c} / 3$. Qualitatively similar results are visible on Fig.4 for SDBBD. For random permutation we increase the decoupling speed and apply a random permutation every 0.025 s. Figure 5 shows one typical random run, illustrating how with high probability the drift effect is reduced and the relative phases converge to a close neighborhood of exact synchronization. Further simulations (not shown) confirm that switching faster make the phases converge closer to each other.

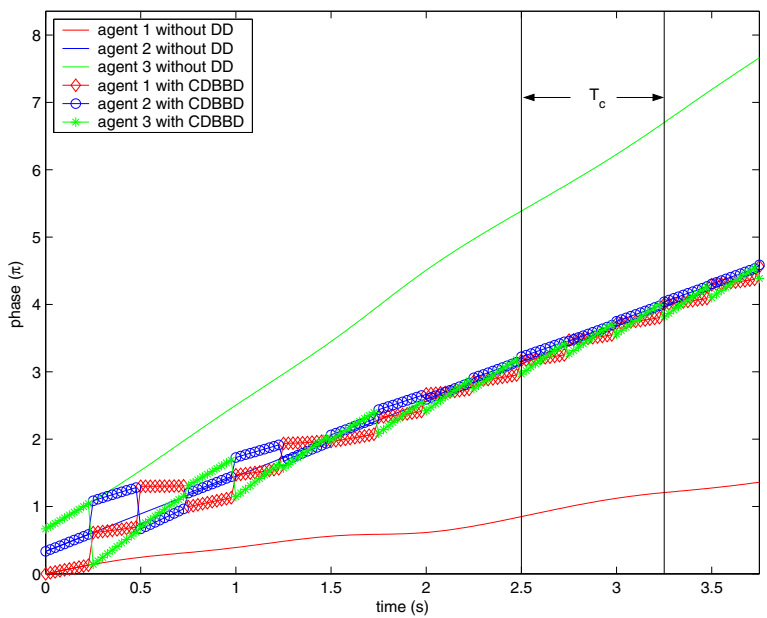

Fig. 3. Phase evolutions with CDBBD, applying $P_{\pi}:(1,2,3) \mapsto(2,3,1)$ every $0.25 \mathrm{~s}$, all-to-all coupling.



Fig. 4. Phase evolutions with SDBBD, applying alternatively $P_{1}$ : $(1,2,3) \mapsto(2,1,3)$ and $P_{2}:(1,2,3) \mapsto(1,3,2)$ every 0.25 s; Kuramoto coupling among oscillators $(1,2)$ and $(2,3)$.

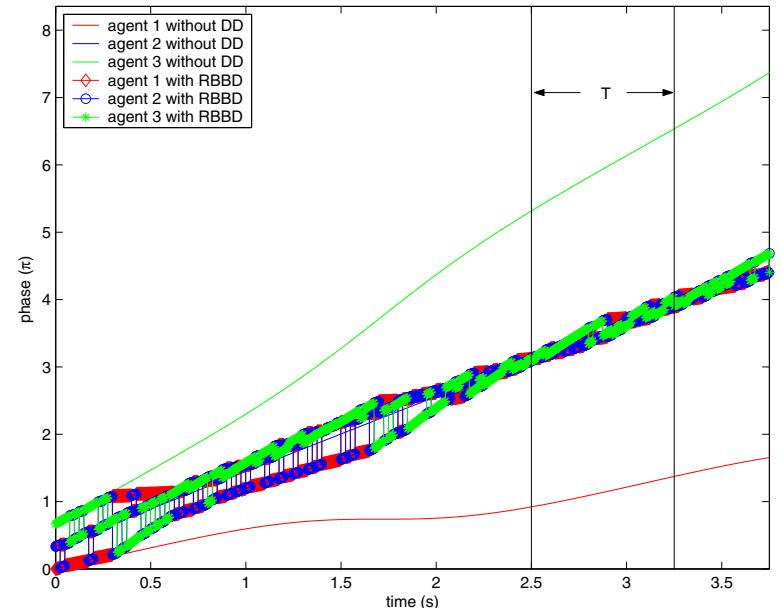

Fig. 5. Phase evolutions with RBBD, applying $P_{1}:(1,2,3) \mapsto(2,1,3)$ or $P_{2}:(1,2,3) \mapsto(1,3,2)$ randomly every $0.025 \mathrm{~s}$; Kuramoto coupling among oscillators $(1,2)$ and $(2,3)$.

\section{CONCLUSION}

This paper proposes a method to counter the effect of nonidentical drift frequencies in coordination control of coupled oscillators, by adding a time-varying control signal inspired by quantum dynamical decoupling. Convergence properties are analyzed in both two-agent and multi-agent situations. In particular, the oscillators can be brought closer to exact synchronization by increasing the speed of the time-varying dynamics. A direct extension of this work would be to adapt the continuous control version, known as Eulerian decoupling, to the multi-agent case. Future work could focus on developing dynamical decoupling solutions for other geometric configuration spaces ubiquitous in robotics, like $S E(2)$ and $S O(3)$, and possibly providing a general theory.

\section{REFERENCES}

[1] Y. Kuramoto. Chemical Oscillations, Waves, and Turbulence. Springer, 1984.

[2] A. T. Winfree. The Geometry of Biological Time. Springer, 1980.

[3] R. Sepulchre, D. A. Paley, and N. E. Leonard. Stabilization of planar collective motion: all-to-all communication. IEEE Trans. Autom. Control, 52(5):811-824, 2007.

[4] A. Jadbabaie, N. Motee, and M. Barahona. On the stability of the Kuramoto model of coupled nonlinear oscillators. Proc. Amer. Control Conference, pages 4296-4301, 2004.

[5] S.H. Strogatz. From Kuramoto to Crawford: exploring the onset of synchronization in populations of coupled nonlinear oscillators. Physica D, 143:120, 2000.

[6] L. Viola, E. Knill, and S. Lloyd. Dynamical decoupling of open quantum systems. Physical Review Letters, 82(12):2417-2421, 1999.

[7] L. Viola and E. Knill. Robust dynamical decoupling of quantum systems with bounded controls. Physical Review Letters, 90(3), 2003.

[8] L.F. Santos and L. Viola. Dynamical control of qubit coherence: random versus deterministic schemes. Physical Review A, 72(6): 062303, 2005.

[9] J. J. Sakurai. Modern Quantum Mechanics. Addison-Wesley, New York, 2007.

[10] L. Mazzarella, A. Sarlette and F. Ticozzi. From consensus to robust randomized algorithms: a symmetrization approach. SIAM J. Control and Optimization, 2013, submitted.

[11] L. Moreau. Stability of multi-agent systems with time-dependent communication links. IEEE Trans. Autom. Control, 50(2):169-182, 2005. 